\title{
Research of Highway Intelligent Vehicles Visual Navigation System
}

\author{
Wang Manzhen \\ Automotive Engineering institute, Jiangxi University of Technology, Nanchang 330098, China
}

Keywords: Intelligent vehicles; Visual navigation; Highway; System development

\begin{abstract}
Intelligent vehicles, also called wheeled mobile robots, are the carriers involving numerous high and new technologies, such as the sensor, database, image processing, fuzzy control, neural network, vehicle dynamics, modern control theory, computer, communication and various programming languages, etc. According to the combination of graphic and image processing and the theory of system development, the paper studies the highway intelligent vehicle visual navigation system.
\end{abstract}

\section{Introduction}

With the development of the society, cars have already become an irreplaceable means of transport in people's lives. However, its attendant problems are also growing, such as environmental pollution, noise and traffic accident, causing uncalculated direct and indirect losses to the society. In recent years, in order to solve the traffic problem, nations all around the world are carrying out researches into the filed of intelligent transportation system (ITS). In highway traffic, relevant researches about intelligent vehicles, as an important part, have been receiving more and more concern and attention from people. Intelligent vehicles, also called wheeled mobile robots, are the carriers involving numerous high and new technologies, such as the sensor, database, image processing, fuzzy control, neural network, vehicle dynamics, modern control theory, computer, communication and various programming languages, etc.

In the constant improvement of intelligent vehicles, a key work is to quickly and accurately use the on-board sensor to obtain the road environment ahead. The commonly used methods include: machine visual technology (also called computer vision or image analysis and understanding, etc.), radar technology, magnetic navigation technology (early adopt paving magnetic blocks in the middle of the road for navigation), high-precision digital maps and global positioning system (GPS), etc. With the rapid development of computer and digital image processing technology, the application of machine visual technology is getting more and more attention from people, which is because compared with other sensors, visual sensor has the following features:

The image has extremely rich information. If breakthrough can be made in image recognition algorithm, it can reduce the operation time of the entire system and improve the accuracy of road test meanwhile. Therefore, it is a very promising research direction to use image processing to understand the road environment. Currently, many researchers have been engaged in researches of this direction.

Visual sensors belong to passive sensors without damaging the road or making great changes to the existing road facilities.

Multiple lanes can be detected with a lot of information acquired. Meanwhile, road test and obstacle recognition can be achieved. 
The maintenance cost is low and real-time video can be provided for experts for afterwards analysis. In addition, the video signals can be transmitted through multiple ways such as radio frequency or microwave, providing the current situation of vehicles for the monitoring center.

Communication with the road or other vehicles is not needed so as to obtain the relatively complete road situation and information about vehicles. In addition, it has low costs without causing pollution to the environment.

Therefore, machine visual technology is very suitable for road detection and tracking. Currently, many domestic and foreign research institutions are engaged in researches of this field. Perfect visual system is made up of two major modules: image acquisition system and image processing system.

The emergence of automotive machine vision can be dated back to the Mars exploration program in the 1960s and 1970s. However, due to the low computer processing capacity at that time, machine vision did not cause much attention. Ever since the 1980s, with the improvement of microprocessor's capacity, the real on-board visual system appeared. The most important factor promoting to the development of on-board machine vision was not the auto industry itself, but the microelectronics technology and computer science. The key factor that on-board machine vision can eventually be practical is whether there will be strong enough computing power and cheap microprocessor, which is the key factor restricting the development of on-board machine vision. In the mid and late 1990s, the microprocessor had already had a enough high performance-price ratio so that machine vision can be applied to commercial vehicles.

Machine vision, also called computer vision, is the most important component part in intelligent vehicle environment sensor system and made up of two main modules: image acquisition system and image processing system. Under the current hardware and software technology condition, although machine vision function is still at the preliminary stage, it has already caused great attention from countries all around the world due to its potential application value. Developed countries like the US, Japan, Germany and France have already invested a lot of manpower and material resources into research, and obtained breakthroughs in some aspect of machine vision in recent years. Machine vision has already been applied to actual vehicles, and shows its importance in application in vehicle safety technology and automation technology. The configuration of machine visual hardware, algorithm of visual processing and on-board machine visual application system based upon the above two are key technologies to improve the reliability of visual navigation.

For road detection technology, the biggest difficulty is still the robustness and real time of algorithm detection. Aiming at the current intelligent vehicle research results based on visual navigation and its deficiencies as well as the analysis of key technologies need to be solved in researches, researches of the following aspects aiming at road detection of highway intelligent vehicles are carried out in this paper:

Use the three characteristics of while line - gray, gray level difference and connectivity at the same time to improve the reliability of the feature points' extracted results.

Divide the road ahead the vehicle into two parts: near vision and far vision. Carry out linear fitting towards white lines in the near vision to obtain information about the heading direction and excursion; analyze white lines in the far vision and carry out linear fitting (curve) when necessary.

In the preliminary detection algorithm and tracking algorithm, use the weighted least square method to obtain the road model that most matches the white line point, and the methods to select weight are not the same. 
Tracking path changes and dynamic updated model in the image sequence, use the time and space continuity of sequence information, and eliminate error detection according to the results of the previous frame.

\section{Theory of Visual Navigation}

Intelligent vehicle navigation research involves a lot of research fields such as machine vision, digital image processing, photography measuring technology, the optimal estimation and sensor fusion, etc.

Machine vision is the science and technology to research computers to simulate biological explicit or macro visual functions. It is the most important component of intelligent care environment perception system with the primary goal as using images to create or restore the real world model and then recognize the real world. While driving cars, more than $90 \%$ of the information that driver obtains is from visual perception, such as road, signs and vehicle condition in the driving environment are all direct or implied visual information. Currently, both China and foreign countries are racing to carry out researches about the basic theories, basic technologies and application of machine vision.

From a broad sense, images are hard copies of the objective world (or objects) through certain optical system, and the generation of images is one of the important links of computer vision. Therefore, before describing and understanding image signals, it is necessary to make clear how "images" are produced, that is, the principles and process of the 3D scenes in the objective world to produce 2D images. Image sampling in this paper is completed through digital camera.

Visual transformation, also called image transformation, is used to project points in the 3D space onto the surface of this visual point and this point. When a spatial point $\mathrm{P}(\mathrm{Xw}, \mathrm{Yw}, \mathrm{Zw})$ is projected on the image surface $S$, there is the point (Xs, Ys). The image place $S$ parallels with the user coordinate system XoY and intersects with the axis $\mathrm{z}$ at $\mathrm{o}$. The image place coordinate system is shown with xsoy, and its distance to the coordinate system's original point is Z2. The distance between the visual point and the user coordinate system's original point is.

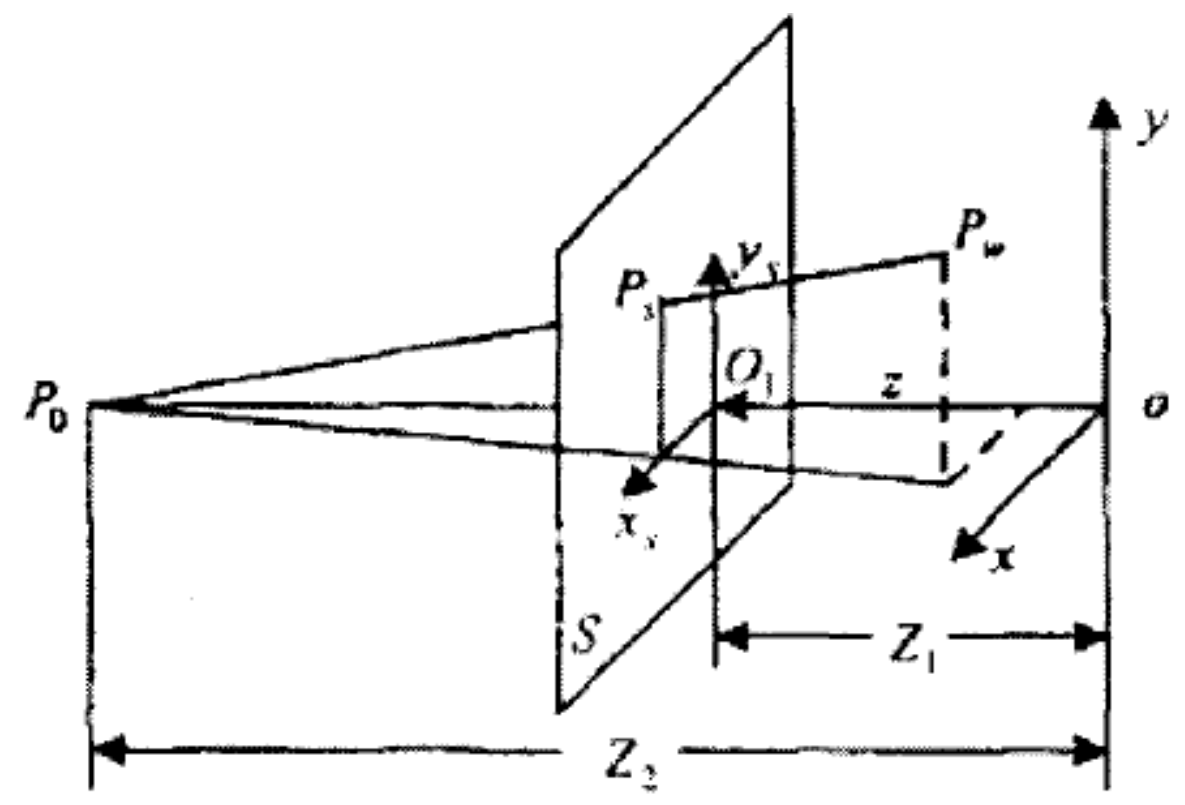

Figure. 1 Perspective Projection Model 
In order to quantitatively describe the process of optical imaging, we first define three coordinate systems: image coordinate system, camera coordinate system and world coordinate system.

Cameras capture images in the form of a standard television signal input into the computer, the computer dedicated digital to analog converter board into a digital image. Each piece of digital images in a computer $\mathrm{MxN}$ array of $\mathrm{M}$ rows and $\mathrm{N}$ columns of the image of each element (called pixels) of the image points of both brightness values (or gray front], if it is a color image, the image on brightness of the pixels will be red, green, and blue represents brightness). 2, the definition of a Cartesian coordinate system in the image $\mathrm{u}$, coordinates $(\mathrm{u}, \mathrm{v})$, respectively, each pixel is the number of columns and rows of pixels in the array. Therefore, (u, v) is the coordinates of the image coordinate system in pixels. Since $(u, v)$ represents a pixel located only a few rows and columns in the array, and not indicative of the position of the pixel in the image with a physical unit, therefore, we need to re-establish the image coordinates in physical units (such as millimeters) Department. The coordinate system to a point within the image 01 as the origin, $x$-axis and $y$-axis parallel to the axis $\mathrm{u}$ respectively as shown in Fig .2, in which (U, V) represents coordinates of the image coordinate system in pixels, $(\mathrm{x}, \mathrm{y})$ represents coordinates millimeters image coordinate system.

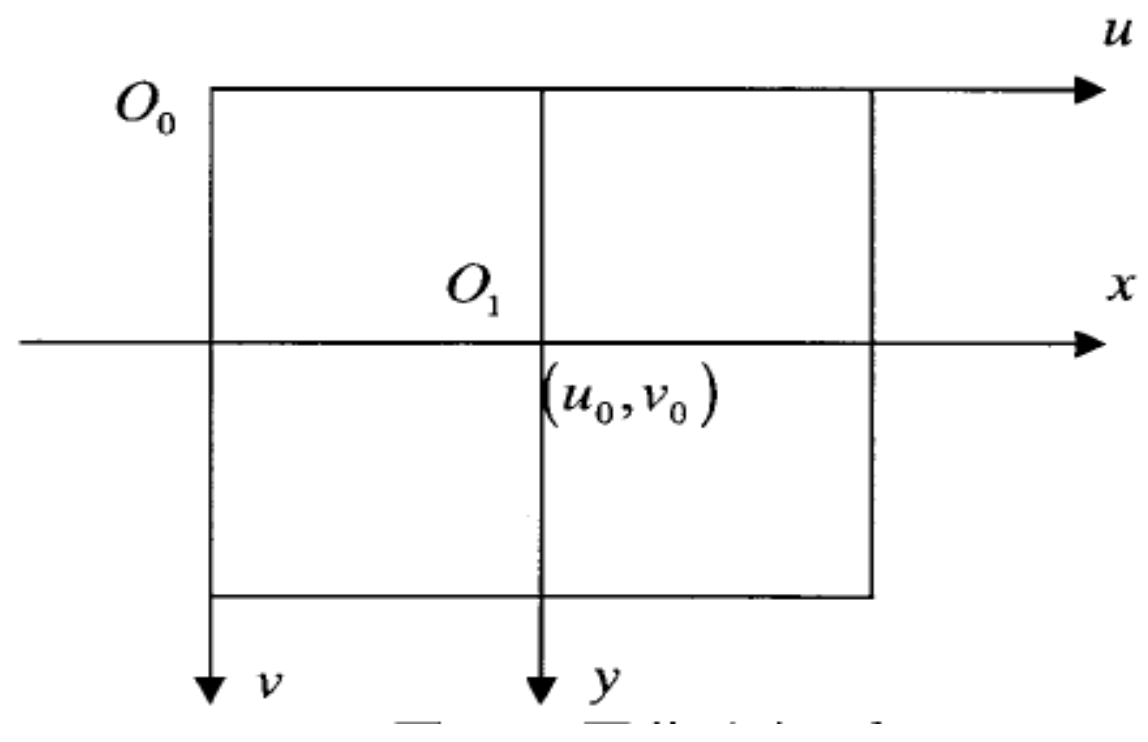

Figure. 2 Image Coordinate System

Images are formed by visual system of the objective world. Generally, images are 2D, and one image can be represented with a $2 \mathrm{D}$ data array $f(x)$, herein, $(x)$ refers to the plane coordinate and $f$ refers to the value of $\mathrm{F}$ of some nature in the image at the point (x). For example, in the grayscale image, f refers to the grayscale, usually corresponding to the observed brightness of the objective scene.

In order to use the computer for image processing, first of all, to carry out continuous image coordinate space and the discrete nature of space, which is sampled and quantized, such a discrete image is a digital image, point sampling quantized called pixels. Road image filtering. The so-called filter is to extract the desired signal from the signals are mixed together in the many. Signal is a function of time or space to pass and carry information. One type of variation of the signal is given, called deterministic signals, you can use physical methods or digital filtering algorithms filtering; another variation of the signal is not established, such as a variety of errors, called random signal, can not use a conventional or suppression filter to extract the signal. It is a random signal Kalman very good manner. Kalman filter to extract the signal from the measuring-related by the algorithm to 
estimate the desired signal, which is estimated to have random response signal is due to white noise excitation, transfer structure (system of equations) between excitation and response are known, Measurement and estimated relationship between the amount of function (measurement equation) is also known. The estimation process utilizes the following information: the system of equations, the measurement equation, the statistical characteristics of white noise, and the statistical characteristics of measurement errors. It is designed in the time domain. White noise excitation system and measurement noise are not required to filter out objects, their statistical characteristics of the process is the information need to use estimates for the Kalman filter is also known as the theory of optimal estimation

\section{Conclusion}

Road detection is an important content of autonomous driving. This paper studies on high-speed roads for autonomous driving car on the road marker line detection. Since perspective road image perspective distortion present in apparently strongly distorted structure of the road, the paper analyzes the camera model, implemented a road image inverse perspective transformation method, and on this basis, to study the lane markings detection.

Lane marking edge detection is an important part of algorithm. Aiming at the characteristics of road structure, this paper analyzes the detailed features of marking lines in global coordinate system images and designs special marking lines to detect the algorithm with good detection effect to global coordinate system images. Through the targeted research of quality changes in the image, this paper proposed the processing method to enhance the marking line and segmentation leap-value.

The extraction of road marking lines is the core of the algorithm and the key to solve this problem based on entire information and model. By referring to the norms of road construction, thus paper analyzes the road structural model and proposes a method to detect and track road marking lines. Track the changes of road in the image sequence and dynamic update the model while using time and space continuum of the sequence information to eliminate wrong detection with the results of the previous frame.

\section{Acknowledgements}

This work was financially supported by the key subject building project (vehicle engineering) of Jiangxi University of Technology.

\section{References}

[1] Wehner R, Michel B, Antonsen P. Visual navigation in insects: coupling of egocentric and geocentric information[J]. The Journal of Experimental Biology, 1996, 199(1): 129-140.

[2] Matsumoto $\mathrm{Y}$, Inaba $\mathrm{M}$, Inoue $\mathrm{H}$. Visual navigation using view-sequenced route representation[C]//Robotics and Automation, 1996. Proceedings., 1996 IEEE International Conference on. IEEE, 1996, 1: 83-88.

[3] Collett T S, Collett M. Memory use in insect visual navigation[J]. Nature Reviews Neuroscience, 2002, 3(7): 542-552.

[4] Gracias N, Santos-Victor J. Underwater video mosaics as visual navigation maps[J]. Computer Vision and Image Understanding, 2000, 79(1): 66-91. 
[5] Wolton R, Moulton W. Method and apparatus for search, visual navigation, analysis and retrieval of information from networks with remote notification and content delivery: U.S. Patent Application 10/112,408[P]. 2002-4-1.

[6] Matsumoto $\mathrm{Y}$, Ikeda $\mathrm{K}$, Inaba $\mathrm{M}$, et al. Visual navigation using omnidirectional view sequence[C]//Intelligent Robots and Systems, 1999. IROS'99. Proceedings. 1999 IEEE/RSJ International Conference on. IEEE, 1999, 1: 317-322.

[7] Kidono K, Miura J, Shirai Y. Autonomous visual navigation of a mobile robot using a human-guided experience[J]. Robotics and Autonomous Systems, 2002, 40(2): 121-130.

[8] Evans Jr J M, Weiman C F R, King S J. Visual navigation and obstacle avoidance structured light system: U.S. Patent 4,954,962[P]. 1990-9-4.

[9] Goedemé T, Tuytelaars T, Van Gool L. Fast wide baseline matching for visual navigation[C]//Computer Vision and Pattern Recognition, 2004. CVPR 2004. Proceedings of the 2004 IEEE Computer Society Conference on. IEEE, 2004, 1: I-24-I-29 Vol. 1.

[10]Hatsopoulos N G, Warren W H. Visual navigation with a neural network[J]. Neural Networks, 1991, 4(3): 303-317. 